\title{
Casimir energy of finite width mirrors: renormalization, self-interaction limit and Lifshitz formula
}

\author{
I. V. Fialkovsky ${ }^{\dagger \ddagger}$, V. N. Markov ${ }^{\llbracket}$ and Yu. M. Pis'mak ${ }^{\ddagger} *$ \\ † ifialk@gmail.com, Instituto de Física, Universidade de São Paulo, São Paulo, Brazil \\ ${ }^{\ddagger}$ Department of Theoretical Physics, Saint-Petersburg State University, Russia \\ ฯ St. Petersburg Nuclear Physics Institute, Russia
}

\begin{abstract}
We study the field theoretical model of a scalar field in presence of spacial inhomogeneities in form of one and two finite width mirrors (material slabs). The interaction of the scalar field with the defect is described with positiondependent mass term. Within this model we derive the interaction of two finite width mirrors, establish the correspondence of the model to the Lifshitz formula and construct limiting procedure to obtain finite self-energy of a single mirror without any normalization condition.
\end{abstract}

Keywords: Casimir energy, QFT, finite width mirrors

\section{Introduction}

The Casimir effect ${ }^{1}$ was original considered for an extremely idealized configuration of electromagnetic field subject to ideal conducting boundary conditions on two parallel plates. Development of both theoretical and experimental techniques required consideration of more realistic systems which would describe real materials, both in their shape and properties. During last decades a number of such approaches were successfully developed, see ${ }^{2}$ for a review.

One of the methods for investigation of (more) realistic situations was initially proposed by Symanzik ${ }^{3}$ and consists of modeling and generalizing the rigid boundary conditions by introducing additional singular-potential terms into the action of the model. This approach was widely explored for the case of delta-type potentials. The essence of this paper is to present the results of elaboration of similar field-theoretical approach in the case of steppotentials. The detailed calculations and discussions are presented in. ${ }^{4,5}$

*The authors gladly acknowledge the support of grants RNP 2.1.1/1575, RFBR 07-0100692. IVF is especially grateful to support of University of Oklahoma and FAPESP. 


\section{Single finite width mirror}

Let us consider a scalar field interacting with a space defect with nonzero volume. Using the Symanzik's approach, ${ }^{3}$ we add to the action an additional mass term being non-zero only inside the defect:

$S=S_{0}+S_{\text {def }}$
$S_{0}=\frac{1}{2} \int d^{4} x \phi(x)\left(-\partial_{x}^{2}+m^{2}\right) \phi(x), \quad S_{\text {def }}=\frac{\lambda}{2} \int d^{4} x \theta\left(\ell, x_{3}\right) \phi^{2}(x)$

where $\partial_{x}^{2}=\partial^{2} / \partial x_{0}^{2}+\ldots+\partial^{2} / \partial x_{3}^{2}$. In the simplest case the defect could be considered as homogenous and isotropic infinite plane layer of the thickness $\ell$ placed in the $x_{1} x_{2}$ plane (so called 'piecewise constant potential')

$$
\theta\left(\ell, x_{3}\right) \equiv\left[\theta\left(x_{3}+\ell / 2\right)-\theta\left(x_{3}-\ell / 2\right)\right] / \ell .
$$

To describe all physical properties of the systems it is sufficient to calculate the generating functional for the Green's functions

$$
G[J]=N \int D \phi \exp \{-S[\phi]+J \phi\}
$$

where $J$ is an external source. The explicit calculation of $G[J]$ is performed with help of integral operators method developed by the authors, see. ${ }^{4,5}$

\subsection{Casimir self-energy and its renormalization}

The Casimir energy density per unit area of the defect $S$ can be presented with the relation $\mathcal{E}=-\frac{1}{T S} \ln G[0]$, here $T$ is the (infinite) time interval and $S$ - the surface area of the defect.

For the case of a single finite-width mirror, as in (1), the Casimir energy is given by a sum of a UV finite, $\mathcal{E}_{\text {fin }}$, and UV divergent, $\mathcal{E}_{\text {div }}$, parts

$$
\begin{gathered}
\mathcal{E}=\mathcal{E}_{\text {fin }}+\mathcal{E}_{\text {div }}, \\
\mathcal{E}_{\text {fin }}=\frac{1}{4 \pi^{2}} \int_{0}^{\infty}\left(\ln \frac{e^{-\ell(E+\sqrt{\rho})}}{4 E \sqrt{\rho} \xi}-\frac{\lambda}{2 E}\left(1-\frac{\lambda}{4 \ell E^{2}}\right)\right) p^{2} d p, \\
\mathcal{E}_{\text {div }}=\frac{\lambda \mu^{4-d}}{2(2 \pi)^{d-1}} \int \frac{d^{d-1} p}{2 E}\left(1-\frac{\lambda}{4 \ell E^{2}}\right),
\end{gathered}
$$

where we used dimensional regularization, and put $\rho=E^{2}+\kappa, \kappa=\lambda / l$, $\xi^{-1}=e^{2 \ell \sqrt{\rho}}(E+\sqrt{\rho})^{2}-(E-\sqrt{\rho})^{2}$. With appropriate redefinitions of the parameters this expression coincides with known results in the literature. ${ }^{7}$

For renormalization of the model at the one-loop level considered here we must add to the action the following field-independent counter-term $\delta S$

$$
\delta S=f \lambda+g \lambda^{2} \ell^{-1},
$$


with bare parameters $f$ and $g$. Within such 'minimal addition' renormalization scheme we obtain for the renormalized Casimir energy

$$
\mathcal{E}_{r}=\mathcal{E}_{f i n}+\lambda f_{r}+g_{r} \lambda^{2} \ell^{-1}
$$

where finite parameters $f_{r}, g_{r}$ must be determined with appropriate experiments, or fixed with normalization conditions. The number of required conditions is dictated by the (in)dependence of the coupling constant $\lambda$ on the slab thickness $\ell$.

\subsection{Dirichlet limit and normalization condition}

Under the change of variables $\lambda \rightarrow \ell \kappa$ two counter-terms in (7) can be effectively combined into a single one $\tilde{g}_{r}$ of mass dimension one

$$
\mathcal{E}_{r}=\mathcal{E}_{\text {fin }}+\kappa \ell \tilde{g}_{r}
$$

One notes that putting $\kappa=-m^{2}$ and taking the $m \rightarrow \infty$ limit effectively converts the system under consideration into a massless scalar field confined between two plates at $x_{3}= \pm \ell / 2$ subject to Dirichlet boundary conditions at the boundaries. We can use this correspondance for fixing $\tilde{g}_{r}$.

The finite part of the Casimir energy (4) in the limit $m \rightarrow \infty$ yields

$$
\mathcal{E}_{f i n}=-\frac{m^{4} \ell}{128 \pi^{2}}+\left(\frac{\pi}{6}-\frac{4}{9}\right) \frac{m^{3}}{4 \pi^{2}}-\frac{\pi^{2}}{1440 \ell^{3}}+O\left(m^{-1}\right)
$$

Now we require that the renormalized energy (8) in this limit lead to the same Casimir pressure as in the case of massless scalar field subject to Dirichlet boundary conditions $\mathcal{P}_{\text {Dir }}=-\frac{\pi^{2}}{480 \ell^{4}}$. Then this condition fixes the renormalization parameter $\tilde{g}_{r}$ of $(8)$

$$
\tilde{g}_{r}=-\frac{m^{2}}{128 \pi^{2}}
$$

Thus, using this Dirichlet limit procedure we are able to collate a particular limit of our results with a well known (unambiguous) physical situation.

\section{Interaction of two finite width mirrors}

Let us consider two plane slabs of thickness $\ell_{1,2}$ interacting over the distance $r$. The action can be written as

$$
\begin{aligned}
S & =S_{0}+S_{d e f}, \quad S_{0}=\frac{1}{2} \int d^{4} x \phi(x)\left(-\partial_{x}^{2}+m^{2}\right) \phi(x) \\
S_{d e f} & =\int d^{3} x\left(\kappa_{2} \int_{-a_{2}-l_{2}}^{-a_{2}} d x_{3} \phi^{2}(x)+\kappa_{1} \int_{a_{1}}^{a_{1}+l_{1}} d x_{3} \phi^{2}(x)\right)
\end{aligned}
$$

here $a_{1}+a_{2} \equiv r$. 
Proceeding along the lines of ${ }^{4,5}$ we get the final expression for the energy

$$
\mathcal{E}_{2 L}=\mathcal{E}_{1}+\mathcal{E}_{2}+\int \frac{d^{3} \vec{p}}{2(2 \pi)^{3}} \log \left[1-e^{-2 E r} \prod_{i=1,2} \kappa_{i} \xi_{i}\left(1-e^{2 \ell_{i} \sqrt{\rho_{i}}}\right)\right] .
$$

Here $\mathcal{E}_{1,2}$ give the self-energy (4) of solitary layers 1, 2 correspondingly. All the notation here follows ones of Sect. 2 with subscript index corresponding to the layer number.

The third term in (12) represents the interaction of two layers and vanishes in the limit $r \rightarrow \infty$. We note that the interaction term is UV finite, and the removal of regularization made in (12) is indeed justified. This is in perfect accordance with general considerations ${ }^{8}$ of the finiteness of Casimir interaction between disjoint bodies.

Basing on the general expression (12) we can calculate the vacuum energy in different limits such as self-pressure of the slab in presence of delta-spike and interaction between two of them. The two delta-spikes limit known in previous literature is also reproduced.

\subsection{Connection to the Lifshitz formula}

Now we consider in (12) the limit of slabs of infinite width separated by finite distance $r$. For the force in such a limit we obtain

$$
\mathcal{F}_{\text {Lif }} \equiv-\frac{\partial \mathcal{E}_{\text {Lif }}}{\partial r}=-\int \frac{d^{3} \vec{p}}{(2 \pi)^{3}} \frac{E}{e^{2 E r} \frac{\left(E+\sqrt{\rho_{1}}\right)\left(E+\sqrt{\rho_{2}}\right)}{\left(E-\sqrt{\rho_{1}}\right)\left(E-\sqrt{\rho_{2}}\right)}-1}
$$

It is straightforward to see that the correspondence with the Lifshitz formula ${ }^{6}$ achieved if we introduce particular dispersion into the interaction of quantum fields with the material defect,

$$
\kappa_{1,2}^{T E}(p)=\left(\epsilon_{1,2}-1\right) p_{0}^{2}, \quad \kappa_{1,2}^{T M}(p)=\left(\frac{1}{\epsilon_{1,2}^{2}}-1\right) \vec{p}^{2}+\left(\frac{1}{\epsilon_{1,2}}-1\right) p_{0}^{2} .
$$

The parameter $\epsilon$ which enters these dispersion relations is to be identified with dielectric permittivity. Summing TE and TM contributions we immediately recover the Lifshitz formula ${ }^{6}$

$$
\begin{gathered}
\mathcal{F}_{\text {Lif }}=-\frac{1}{4 \pi^{2}} \int_{0}^{\infty} d p_{0} \int_{0}^{\infty} d p^{2} E\left(d_{T E}^{-1}+d_{T M}^{-1}\right) \\
d_{(T E, T M)}=e^{2 E r} \frac{\left(E+\sqrt{\rho_{1}^{(T E, T M)}}\right)\left(E+\sqrt{\rho_{2}^{(T E, T M)}}\right)}{\left(E-\sqrt{\rho_{1}^{(T E, T M)}}\right)\left(E-\sqrt{\rho_{2}^{(T E, T M)}}\right)}-1
\end{gathered}
$$

with $\rho_{1,2}^{(T E, T M)}=E^{2}+\kappa_{1,2}^{(T E, T M)}(p)$. 


\subsection{Self-interaction limit}

For $\kappa_{2}=\kappa_{1}$ the limit $\ell \rightarrow \infty$ taken in the two-slab action (11) reproduces the single-slab action (1) subject to the substitution $\ell \rightarrow r, m^{2} \rightarrow m^{2}+\kappa_{1}$, $\lambda \rightarrow-\kappa_{1} r$. One would expect that the Casimir energy of two slabs in this limit also reproduces the result for a single finite-width mirror.

However, one finds that $\mathcal{F}_{\text {Lif }}$ (13), being UV finite in this limit, differs from UV divergent $r$-derivative of (4) $\partial \mathcal{E} / \partial r$ by

$$
\Delta=-\frac{\mu^{4-d}}{2(2 \pi)^{d-1}} \int d^{d-1} \vec{p}\left(E-\sqrt{E^{2}+\kappa}\right)
$$

Yet, this discrepancy does not signal any inconsistency. In Sec. 2 we presented a derivation of the Casimir self-energy of a single slab. It is known that in general the self-interactions of Casimir type do possess divergencies depending on the geometrical properties of the system. This fact is reflected in the presence of the counter terms (6).

On the other hand, it is well known, ${ }^{8}$ that Casimir interaction of two disjoint bodies is always free of divergencies depending on the distance between them. This very system was considered in this section, and the force (13) between two distinct slabs was found to be finite and unambiguous. Consequently, the limit of infinite width of the slabs which recover the selfpressure of a single body, does not bring any divergencies or ambiguities.

Requiring that (8) coincides with (13) we can fix the counter terms $\tilde{g}_{r}$ and collate the two approaches. One finds that this condition fixes the same value for $\tilde{g}_{r}$ as one elaborated in Sec. 4 and given by (10).

Thus, we establish a new divergence free approach to calculate the selfpressure of a single finite width slab which lead to the same result as one obtained imposing a physically motivated normalization condition.

\section{References}

1. H. B. G. Casimir, Proc. Kon. Nederl. Akad. Wet. 51 (1948) 793.

2. M. Bordag, U. Mohideen, and V. M. Mostepanenko, New developments in the Casimir effect, Phys. Rept., 353:1-205, 2001; arXiv: quant-ph/0106045.

3. K. Symanzik, Nucl. Phys. B 190, 1 (1981).

4. I. Fialkovskiy, Y. Pis'mak, V. Markov, arXiv:0907.1058 [hep-th].

5. I. Fialkovskiy, Y. Pis'mak, V. Markov, Phys. Rev. D79 (2009) 028701, arXiv:0812.2430v1 [hep-th].

6. E. M. Lifshitz, Zh. Eksp. Teor. Fiz. 29, 94 (1956),

7. M. Bordag, J. Phys. A 28, 755 (1995). R. A. Konoplya, D. V. Vassilevich, JHEP 01 (2008) 068, arXiv:0712.0360

8. T. Emig, et al., Phys. Rev. Lett. 99 (2007) 170403, arXiv:0707.1862. 